\title{
Contribution à la connaissance du comportement et de la biologie des larves de marbré Lithognathus mormyrus (sparides) en élevage
}

\author{
M. KENTOURI et P. DIVANACH \\ Station de biologie marine et lagunaire, \\ quai de Bosc prolongé, F 34200 Sète
}

\section{Résumé}

Des larves de marbré Lithognathus mormyrus sont élevées depuis l'éclosion jusqu'au stade alevin dans des bassins de grand volume.

Sous des conditions d'alimentation polyspécifiques polydimensionnelles incluant des ciliés pélagiques marins, les individus présentent une croissance et une survie élevées et une évolution comportementale voisine de celle décrite pour d'autres sparidés (KentourI \& Divanach, 1982).

Le bol alimentaire est toujours diversifié, mais dominé par 4 catégories de proies qui sont successivement : de 2,9 à $3,5 \mathrm{~mm}$, les ciliés et les véligères; de 4 à $7 \mathrm{~mm}$, les rotifères; au-delà de $7 \mathrm{~mm}$, les crustacés.

Lorsque les ciliés ne sont pas représentés dans la ration, l'entrée dans la phase trophique est perturbée, la croissance est faible ou nulle, la mortalité très élevée et les survivants sujets à une phase critique. Après une période de prospection supranormale, l'activité diminue, le bol alimentaire se restreint puis les animaux reprennent les postures de repos caractéristiques des prélarves et meurent.

\section{Introduction}

Si l'aire de répartition géographique du marbré Lithognathus mormyrus est maintenant bien définie (Moreau, 1881 ; Cadenat, 1937 ; Lozano Y Rey, 1952 ; SMITH, 1953 ; Lozano CaBo, 1969), la biologie et l'écologie de l'espèce sont encore très mal connues. La sexualité fait l'objet de controverses (SuAU, 1955, 1970 ; Lissia-FraU, 1968). Les stades embryonnaires et larvaires, décrits en élevage (Divanach \& KENTOURI, à paraître), ne le sont que partiellement en milieu naturel (RANZI, 1934 ; VARAGNOLO, 1963). Les données concernant la susceptibilité aux facteurs externes sont ponctuelles (Heldt, 1926 ; Phisalix, 1931 ; Zuniga \& Suau, 1967). Le régime alimentaire n'est connu que chez les juvéniles et les adultes (Suau, 1970 ; Froglia, 1977). D'une façon générale, les principales lacunes biologiques sont relatives aux larves et aux alevins.

Le présent travail, effectué sous des conditions d'élevage en grand volume, nous permet de rapporter des éléments relatifs au comportement général et trophique et à la biologie des très jeunes stades. 


\section{Matériel et méthode}

Les gamètes sont prélevés par pression abdominale sur des géniteurs sauvages naturellement fluents. La fécondation artificielle se fait en milieu hum:de. Les 18 premières heures de l'incubation se déroulent dans une cuve cylindrique de 20 litres en milieu fermé légèrement brassé par aération puis les œufs sont récoltés et répartis dans les bacs définitifs où se fait l'éclosion.

Deux expériences d'élevage larvaire sont effectuées :

- la première, voisine de la méthode extensive, est réalisée dans un milieu stagnant qui subit sans aucune protection toutes les fluctuations nycthémérales et climatiques (bassin extérieur de $5 \mathrm{~m}^{3}$ et $1 \mathrm{~m}$ de profondeur). L'alimentation est fournie par un bloom endogène de ciliés préservé par la faible densité initiale en larves $(4,7 / 1)$ et par un apport exogène de plancton vivant pêché en milieu naturel et tamisé entre 71 et 360 microns pendant le premier mois et non trié ultérieurement;

- la seconde, plus proche de conditions d'élevage intensives, se déroule dans un bac intérieur de $7 \mathrm{~m}^{3}$ et $1 \mathrm{~m}$ de profondeur, éclairé en permanence grâce à des tubes fluorescents Philips Coll white $\left(50 \mathrm{~W} / \mathrm{m}^{2}\right)$ et ne subit aucune des fluctuations thermiques $\left(\mathrm{T}^{\circ}=20^{\circ} \mathrm{C}\right)$ ou salines $(37 \mathrm{p} .1000)$ liées aux intempéries. L'alimentation est presque exclusivement exogène de par la disparition rapide du bloom endogène de ciliés lié à la forte densité initiale en larves $(17,4 / 1)$.

L'élevage de post-larves est effectué dans un bac extérieur de $23 \mathrm{~m}^{3}$ en circuit renouvelé à 10 p. 100 /jour. L'alimentation y est fournie par un bloom endogène de copépodes, caprelles et annélides préservé par la très faible densité d'individus $(18,8$ poissons $\left./ \mathrm{m}^{3}\right)$.

La méthode d'étude consiste en un suivi des critères ci-après définis :

- le comportement des larves dans les bassins fait l'objet d'une observation directe d'au moins 5 heures par jour. Les schémas de distribution spatiale, la forme des essaims, le mode de prospection alimentaire, la forme des trajectoires sont notés sur des fiches quotidiennes complétées dans le cas de l'essai extérieur par des renseignements climatiques (température de l'eau, couverture nuageuse, intempéries, position du soleil, moment de la journée). Chaque jour, 5 à 20 larves sont observées isolément pendant 20 à 500 secondes jusqu'à ce qu'elles soient perdues de vue. Toutes leurs actions sont enregistrées sur magnétophone portable puis dépouillées et minutées au laboratoire. Les distances parcourues sont estimées grâce à des repères placés sur le bassin. La durée des périodes de repos et d'arrêt, la vitesse de nage, la durée et la fréquence d'apparition de chacune des étapes du schéma trophique, les réactions aux stress directs (toucher par aiguille) ou indirects (vibrations appliquées sur les parois, vagues, turbulences) sont déterminés.

La croissance et les contenus stomacaux sont déterminés par mensuration et dissection sous loupe binoculaire d'individus anesthésiés au phénoxy-2 éthanol à la dose de $0,5 \mathrm{ml} / 1$. Les proies ingérées sont comptées, identifiées et mesurées selon leurs 2 dimensions maximales;

- dans le bol alimentaire, les ciliés se présentent sous forme d'une pâte verte à jaune-vert (lorsqu'elle est mélangée à des trochophores) et qui ressemble à des flocs algaux. L'identification de Favella est aisée de par sa lorica de grande taille non digérée, 
mais il n'en va pas de même pour Stenosemella et Amphorella dont la lorica est fragile et pour Strobilidium qui est un cilié non loriqué. Lorsque leur identification était impossible, le nombre ingéré a été déduit du volume d'amalgame de petits ciliés (estimé selon ses 3 dimensions) divisé par le volume unitaire moyen de ces proies. Ce mode de calcul ne tenant pas compte des pertes par digestion sous-estime certainement le rôle des ciliés dans l'alimentation. Le poids unitaire des proies est estimé par leur volume multiplié par un facteur de densité égal à 1,03 ;

Le bilan total des observations se résume de la façon suivante : durant les 37 premiers jours, 89 prélarves et 435 larves ont été sacrifiées, 1836 proies ont été extraites du tube digestif puis inventoriées et répertoriées. 199 prélarves et 93 larves saines ont été observées individuellement pendant des temps moyens respectifs de 57,3 et 90,8 sec (soit un total de 5,5 heures) afin de définir l'évolution des activités de nage et de chasse de l'espèce dans les conditions précitées. 40 larves anormales ou malades observées de la même façon durant 1,6 heure ont permis de définir les limites de l'éthogramme.

\section{Résultats}

\section{A. Comportement des prélarves}

Pendant ce stade qui dure 4 jours à $20^{\circ} \mathrm{C}$, le comportement des marbrés est proche de celui décrit pour le sar, le sargue et la daurade (Kentouri \& Divanach, 1982) et les différences dans les conduites des 2 lots de poissons étudiés sont faibles. A l'éclosion, les animaux mesurent $1,7 \mathrm{~mm}$ et sont hyponeustoniques. A partir de $2 \mathrm{~mm}$ ( 5 heures) ils deviennent pélagiques et se dispersent dans le milieu de façon différente dans les essais 1 et 2 . La répartition semble régie par le facteur lumière (tabl. 1) car sa variabilité pendant la journée est minime en bac intérieur et élevée en bac extérieur. Le comportement moteur se résume à une alternance de bouffées d'activité d'une à $20 \mathrm{sec}$. et de périodes de repos dont la durée diminue au cours du temps pour finir par une nage ininterrompue vers l'âge de 5 jours (fig. 1 et 2 ). Le mode de propulsion par vibration caudale conduit à des trajectoires spiralées et contournées jusqu'à l'apparition des pectorales qui, stabilisant la position du corps, rendent la nage de plus en plus rectiligne. Les mouvements se font dans le sens vertical les 2 premiers jours, en oblique le $3^{\mathrm{e}}$ jour et dans le plan horizontal à partir du $4^{\mathrm{e}}$ jour. La sensibilité mécanique des prélarves est importante. Les stress indirects diffus de type vibration ou vagues, conduisent à une migration verticale passive vers le fond. Les agressions directes amènent une fuite active qui ne devient orientée dans le sens opposé au stress qu'après le $2^{\circ}$ jour. La réitération des réponses augmente avec l'âge et la fatigue conduit toujours à une reprise de la position de repos tête en bas suivie d'une chute verticale passive.

\section{B. Comportement des larves}

A partir du $4^{e}$ jour qui correspond à l'entrée dans la vie trophique $(2,9 \mathrm{~mm})$, les deux essais ont présenté une évolution différente tant sur le plan de la croissance, de la survie que de l'expression comportementale (tabl. 2, 3 et 4) se résumant de la façon suivante :

Essai 1 : maturation comportementale, croissance rapide dès le premier jour, mortalité faible. 

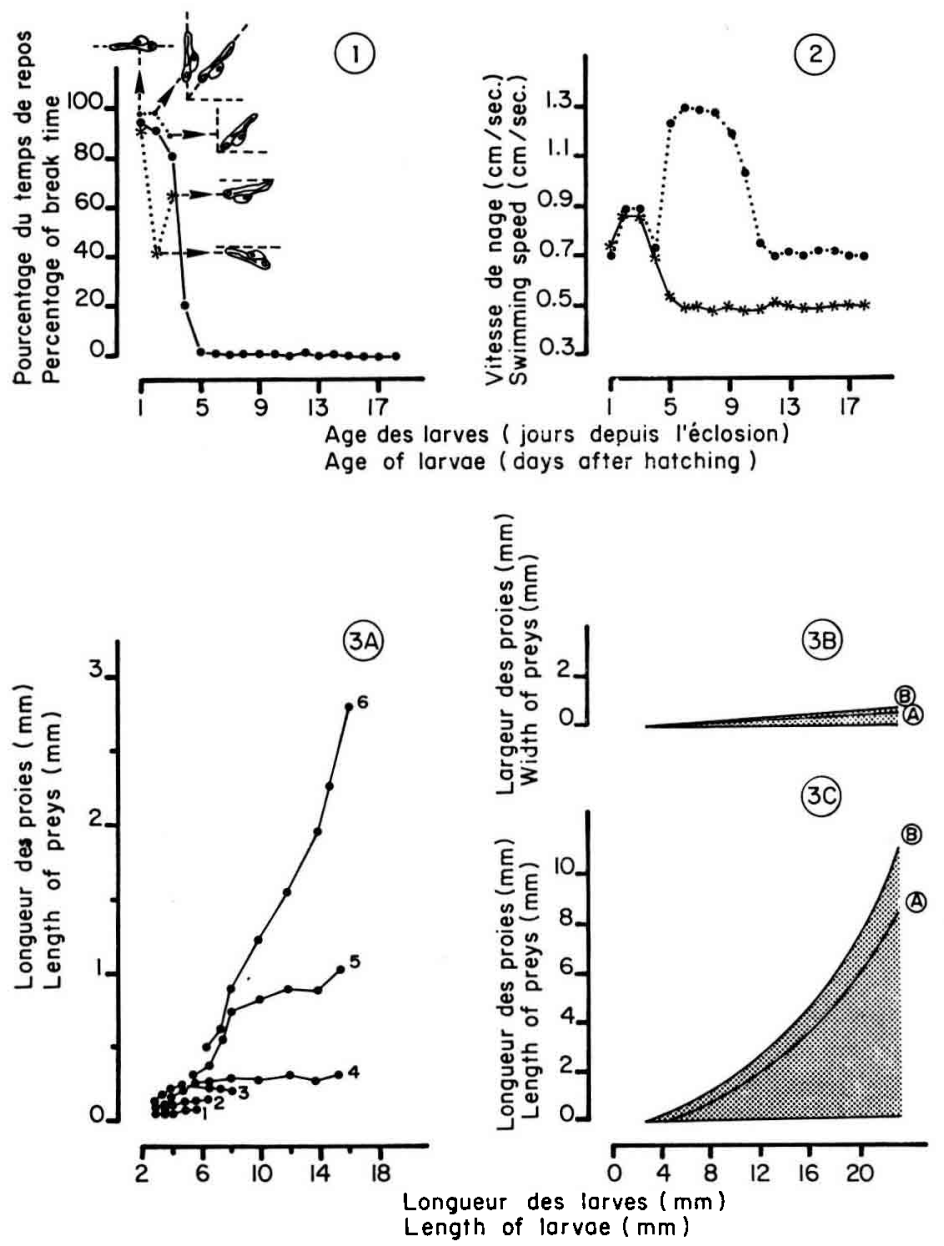

Fig. 1

Evolution du pourcentage du temps et des postures de repos en fonction de l'âge des larves et de leur localisation.

Larval rest postures and length according to age and location.

(.) : Extrême surface - Surface; (*) : Pleine eau - Mid water; (•) : Valeurs moyennes Average.

FIG. 2

Evolution de la vitesse de nage de $\mathrm{L}$. mormyrus pendant les essais $E_{1}(*)$ et $E_{z}(\bullet)$.

Evolution of $\mathbf{L}$. Mormyrus swimming speed during trials $E_{1}(*)$ and $E_{2}(\bullet)$.

FIG. $3 \mathrm{~A}$

Evolution de la longueur moyenne de quelques types de proies dans le contenu digestif des larves de marbré en fonction de leur taille.

Mean length of some prey in the gut.

1 : Trochophores et petits ciliés - Trochophores and little ciliates; 2 : Véligères de lamellibranches et Favella - Veligers and Favella; 3 : nauplii de copépodes et de balanes Copepods and barnacles nauplii; 4 : Brachionus plicatilis d'origine marine et lagunaire B. plicatilis from sea and lagoons; 5 : Copépodites et copépodes-Copepodits and copepods; 6: Artemia salina - A. salina.

FIG. 3 B

Evolution de la gamme de largeur des proies dans le tube digestif en fonction de la longueur des larves.

Prey width range in the gut according to larval size.

A : Limite maximale pour la larve de $S$. auratus (Kentouri \& Divanach, 1982) - Maximum range for $\mathrm{S}$. auratus; $\mathrm{B}$ : Limite maximale pour L. mormyrus - Maximum range for L. mormyrus. 


\section{TABleau 1}

Répartition des larves de marbré au cours du temps dans les bacs extérieur et intérieur.

Distribution with time of stripped sea bream larvae in outdoor and indoor tanks.

\begin{tabular}{|c|c|c|}
\hline $\begin{array}{l}\text { Taille des individus } \\
(\mathrm{mm}) \\
\text { et âge (heures) } \\
\text { après l'éclosion } \\
\end{array}$ & Météorologie & Répartition \\
\hline $\begin{array}{l}1,7-2 \mathrm{~mm} \\
0-5 \mathrm{~h}\end{array}$ & Beau temps & Hyponeustonique \\
\hline $2,0-2,6 \mathrm{~mm}$ & Beau temps & $\begin{array}{ll}\text { Bac extérieur: } & 80 \text { p. } 100 \text { de la population dis- } \\
& \text { persée entre } 0 \text { et } 50 \mathrm{~cm} \text {, } \\
\text { le reste, de } 50 \mathrm{~cm} \text { au fond } \\
\text { Bac intérieur : } & 80 \text { p. } 100 \text { de la population dis- } \\
& \text { persée entre } 0 \text { et } 5 \mathrm{~cm} \text {, } \\
& \text { le reste de } 5 \text { à } 20 \mathrm{~cm}\end{array}$ \\
\hline $2,6-2,9 \mathrm{~mm}$ & Beau temps & 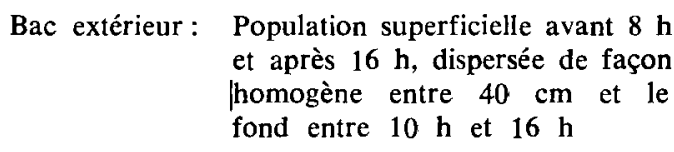 \\
\hline $24-72 \mathrm{~h}$ & Beau temps & $\begin{array}{ll}\text { Bac intérieur: } & 80 \text { p. } 100 \text { de la population dis- } \\
& \text { persée entre } 0 \text { et } 30 \mathrm{~cm} \text {, le reste } \\
\text { dans la tranche inférieure }\end{array}$ \\
\hline \multirow[t]{3}{*}{$2,9-4,5 \mathrm{~mm}$} & $\begin{array}{l}\text { Beau temps } \\
\text { Temps nuageux }\end{array}$ & $\begin{array}{ll}\text { Bac extérieur: } & \text { Dispersion homogène de } 0 \text { à } \\
& 60 \mathrm{~cm} \text { avant } 10 \mathrm{~h} \text { et après } 16 \mathrm{~h} \text {, } \\
& \text { essaim superficiel entre } 10 \mathrm{~h} \text { et } \\
& 16 \mathrm{~h}\end{array}$ \\
\hline & & $\begin{array}{ll}\text { Bac extérieur : } & \text { Dispersion homogène de } 0 \text { à } \\
& 60 \mathrm{~cm} \text { toute la journée }\end{array}$ \\
\hline & & $\begin{array}{ll}\text { Bac extérieur: } & \text { Dispersion homogène de } 0 \text { à } \\
& 60 \mathrm{~cm} \text { toute la journée }\end{array}$ \\
\hline $4,5-13,5 \mathrm{~mm}$ & Temps indifférent & $\begin{array}{l}\text { Bac extérieur et bac intérieur : Dispersion homo- } \\
\text { gène de } 20 \mathrm{~cm} \text { à } 90 \mathrm{~cm}\end{array}$ \\
\hline$>13,5 \mathrm{~mm}$ & Temps indifférent & $\begin{array}{l}\text { Bac extérieur et bac intérieur : Animaux se dé- } \\
\text { plaçant en petits groupes princi- } \\
\text { palement au niveau du fond. } \\
\text { Montée en surface lors de la dis- } \\
\text { tribution, fuite vers le fond et } \\
\text { enfouissement dans le sédiment } \\
\text { lorsqu'ils sont effrayés (>à } \\
20 \mathrm{~cm} \text {.) }\end{array}$ \\
\hline
\end{tabular}

(Suite des légendes de la page 138)

FIG. $3 \mathrm{C}$

Evolution de la gamme de longueur des proies dans le tube digestif en fonction de la longueur des larves.

Prey length range in the gut according to larval size.

A : Limite maximale pour $S$. auratus (Kentouri \& Divanach, 1982) - Maximum range for S. auratus ; B : Limite maximale pour L. mormyrus - Maximum range for L. mormyrus. 
TABLEAU 2

Comparaison du contenu stomacal des larves dans les essais 1 et 2.

Comparison of larval gut content during trials $l$ and 2.

\begin{tabular}{|c|c|c|c|c|}
\hline \multicolumn{2}{|c|}{ Classe de taille - Range of size } & $2,9-3,2$ & $3,2-3,5$ & $3,4-4$ \\
\hline \multicolumn{2}{|c|}{ Essais - Trials } & $\mathrm{E}_{1} / \mathrm{E}_{2}$ & $\mathrm{E}_{1} / \mathrm{E}_{2}$ & $\mathrm{E}_{1} / \mathrm{E}_{\mathrm{g}}$ \\
\hline \multirow{3}{*}{$\begin{array}{l}\text { Contenu stomacal } \\
\text { moyen } \\
\text { Average gut content }\end{array}$} & $\begin{array}{l}\text { Nombre de proies/larve } \ldots \ldots \\
\text { Number of preys/larva }\end{array}$ & $2,4 / 0,93$ & $4,7 / 6,9$ & $8,3 / 4,3$ \\
\hline & $\begin{array}{l}\text { Longueur moyenne des proies } \\
(\mu \mathrm{m}) \ldots \ldots \ldots \ldots \ldots \ldots \ldots \ldots \\
\text { Average length of preys }(\mu \mathrm{m})\end{array}$ & $121 / 118$ & $131 / 136$ & $159 / 130$ \\
\hline & $\begin{array}{l}\text { Largeur moyenne des proies }(\mu \mathrm{m}) \\
\text { Average width of preys }(\mu \mathrm{m})\end{array}$ & $69 / 85$ & $84 / 92$ & $94 / 93$ \\
\hline $\begin{array}{l}\text { Fréquence en nom- } \\
\text { bre des proies dans } \\
\text { l'estomac }\end{array}$ & 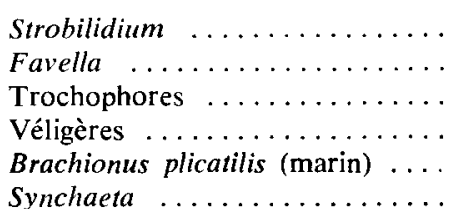 & $\begin{array}{r}33,7 / 14,3 \\
24,1 / 14,3 \\
8,4 / 21,4 \\
12 / 42,9 \\
4,8 / 7,1 \\
7,2 / 0\end{array}$ & $\begin{array}{c}9,6 / 0 \\
1,6 / 0 \\
5,3 / 16,5 \\
28,7 / 49,5 \\
20,2 / 27,8 \\
8,5 / 0\end{array}$ & $\begin{array}{c}0 / 0 \\
6,6 / 0 \\
0,5 / 53,9 \\
37,4 / 38,5 \\
27,8 / 7,7 \\
9,6 / 0\end{array}$ \\
\hline $\begin{array}{l}\text { Number frequency } \\
\text { of preys in the gut }\end{array}$ & $\begin{array}{l}\text { Nauplii de copépodes } \ldots \ldots \ldots \\
\text { Larves d'annélides } \ldots \ldots \ldots \ldots \\
\text { Cypris } \ldots \ldots \ldots \ldots \ldots \ldots \ldots \\
\text { Copépodites } \ldots \ldots \ldots \ldots \ldots \ldots\end{array}$ & 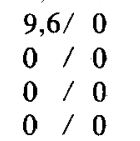 & $\begin{array}{ll}6,4 & 4,1 \\
0 & 0 \\
0 & 0 \\
5,3 / 2,1\end{array}$ & $\begin{array}{cc}10,1 / 0 \\
2 / 0 \\
3 / 0 \\
0,5 / 0\end{array}$ \\
\hline
\end{tabular}

\section{TABlEAU 3}

Comparaison de l'activité de chasse des larves de marbré dans les bassins $E_{1}$ et $E_{2}$. Comparison of stripped sea bream hunting activity during trials 1 and 2.

\begin{tabular}{|c|c|c|c|c|c|c|c|}
\hline $\begin{array}{l}\mathrm{Nb} / \text { jour/larve } \\
\mathrm{Nb} / \text { day/larva } \\
\text { Jours après } \\
\text { l'éclosion }\end{array}$ & $\begin{array}{l}\text { Observa- } \\
\text { tions } \\
\text { Observa- } \\
\text { tions } \\
\text { (a) }\end{array}$ & $\begin{array}{c}\text { Aban- } \\
\text { dons } \\
\text { Surren- } \\
\text { ders } \\
\text { (b) }\end{array}$ & $\begin{array}{l}\text { Position- } \\
\text { nements } \\
\text { S. posi- } \\
\text { tions } \\
\text { (c) }\end{array}$ & $\begin{array}{l}\text { Aban- } \\
\text { dons } \\
\text { Surren- } \\
\text { ders } \\
\text { (d) } \\
\end{array}$ & $\begin{array}{l}\text { Attaques } \\
\text { manquées } \\
\text { Attack } \\
\text { failure } \\
\text { (e) } \\
\end{array}$ & $\begin{array}{l}\text { Régurgi- } \\
\text { tations } \\
\text { Regurgi- } \\
\text { tations } \\
\text { (e) }\end{array}$ & $\begin{array}{l}\text { Inges- } \\
\text { tions } \\
\text { Inges- } \\
\text { tions } \\
(\mathrm{e}) \\
\end{array}$ \\
\hline latching & $\mathrm{E}_{1} / \mathrm{E}_{2}$ & $\mathrm{E}_{\mathrm{l}} / \mathrm{E}_{\mathbf{2}}$ & $\mathrm{E}_{1} / \mathbf{E}_{2}$ & $\mathrm{E}_{1} / \mathrm{E}_{2}$ & $\mathrm{E}_{\mathrm{t}} / \mathrm{E}_{2}$ & $\mathrm{E}_{1} / \mathrm{E}_{2}$ & $E_{1} / E_{2}$ \\
\hline $4^{e}$ & $279 / \quad 0$ & $123 /-$ & $106 /-$ & $0 /$ & $71 /-$ & $0 /-$ & $53 /-$ \\
\hline $6^{\circ}$ & $47 / 201$ & $32 / 101$ & $16 / 101$ & $0 / 25$ & $0 / 25$ & $0 / 25$ & $16 / 25$ \\
\hline $9^{e}$ & $170 / 1004$ & $136 / 753$ & $34 / 279$ & $0 / 112$ & $0 / 56$ & $0 / 0$ & $34 / 112$ \\
\hline $11^{\mathrm{e}}$ & $111 / 325$ & $55 / 252$ & $55 / 72$ & $0 / \quad 0$ & $0 / 0$ & $0 / 0$ & $55 / 72$ \\
\hline $15^{\circ}$ & $571 / 739$ & $457 / 702$ & $114 / 37$ & $0 / 0$ & $0 / 0$ & $0 / 0$ & $114 / 37$ \\
\hline
\end{tabular}

Les données sont exprimées en nb/jour/larve et calculées sur la base de $16 \mathrm{~h}$ de jour dans l'essai $E_{1}$ et de $24 \mathrm{~h}$ de jour dans l'essai $E_{2} ; a$ : après découverte ; $b$ : après observation ; c : après observation; d : après positionnement; e : après attaque - Data are given in No./day/larva and are calculated on the basis of $16 \mathrm{~h}$ lighting/day in trial $l$ and $24 \mathrm{~h}$ lighting/day in trial 2; $a$ : after detection; $b$ : after observation; $c$ : after observation; $d:$ after $S$-position; $e:$ after attack. 
ETHOLOGIE DES LARVES DE MARBRE

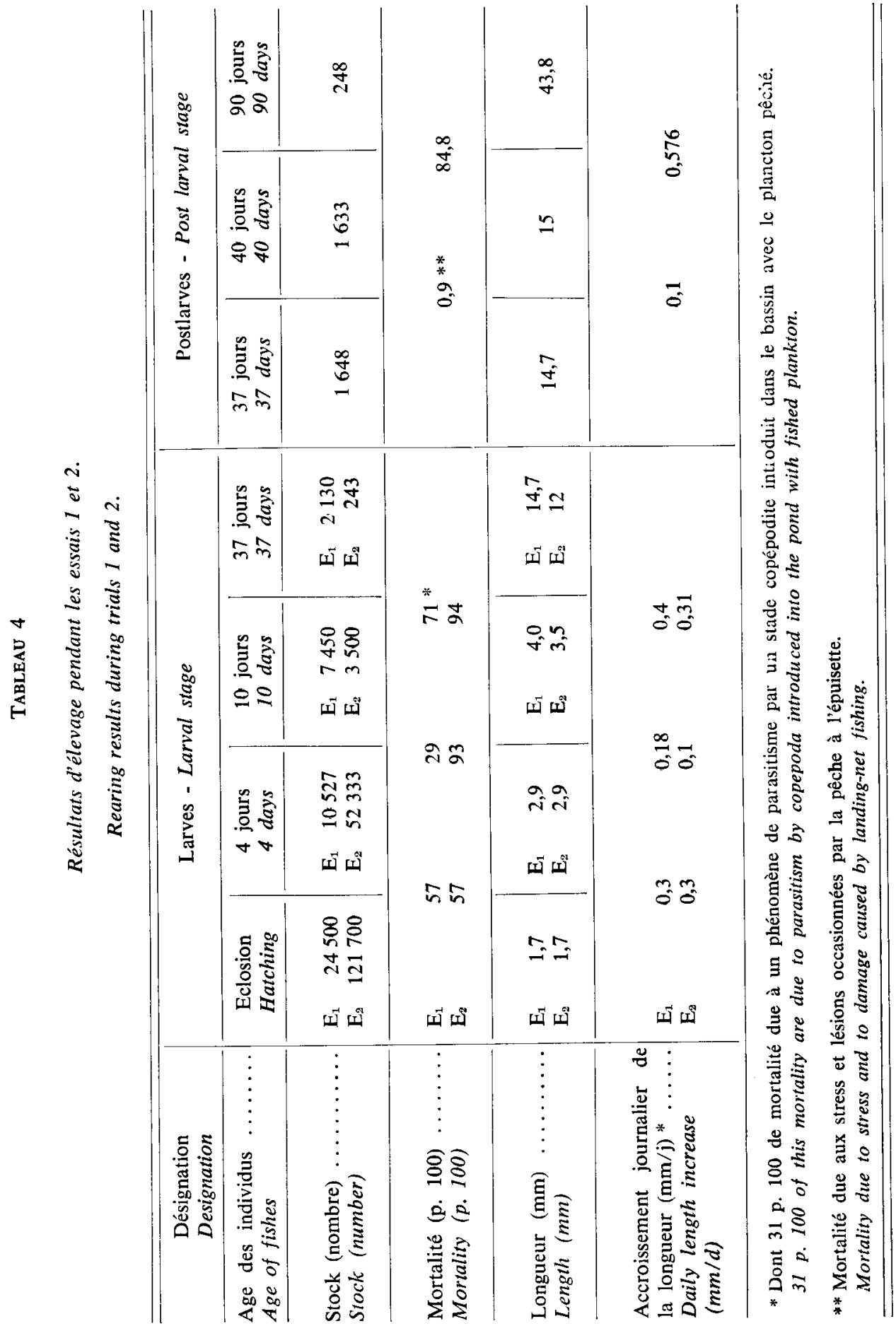


Essai 2 : anomalie comportementale, stagnation de la croissance, mortalité très élevée entre le $9^{\mathrm{e}}$ et le $13^{\circ}$ jour.

\section{Maturation comportementale au cours de l'essai $E_{1}$}

\section{a) Comportement trophique}

Tout comme chez d'autres membres de la famille (Kentouri \& Divanach, 1982), le schéma alimentaire du marbré est constitué d'une phase de prospection, d'une phase de positionnement et visée suivie d'abandon ou de détente, elle-même aboutissant à l'ingestion, la régurgitation ou l'échec. La maîtrise de l'attaque semblerait passer par un apprentissage postural d'une part et d'autre part une accélération des séquences en fonction de la taille des individus, ainsi que de la taille et de la vitesse de nage des proies. Le premier jour, les postures de visée sont en virgule, 33 p. 100 des attaques échouent et 69 p. 100 des proies observées sont abandonnées principalement pour des raisons de durée de positionnement et de fuite des planctontes. Dès le $2^{\mathrm{e}}$ jour d'alimentation, l'acte consommatoire prend les caractéristiques d'un arc réflexe inférieur à une seconde. Les postures de visées en $S$ deviennent quasiment générales mais certaines formes planctoniques petites ou peu mobiles (Synchaeta, véligères) sont parfois attaquées après une ébauche de positionnement et une détente instantanée. La transition entre ces 2 formes d'attaque semble dépendre de la mobilité et de la taille des proies et traduire un phénomène adaptatif. Vers $10 \mathrm{~mm}$, l'enchaînement de l'ensemble des séquences de chasse est très rapide, mais au-delà de $15 \mathrm{~mm}$, elles ne sont plus employées que sporadiquement pour des grosses proies et le mode d'ingestion principal devient l'aspiration buccale.

Tout comme le schéma trophique, le mode de prospection alimentaire évolue avec la taille des animaux selon le récapitulatif suivant :

2,9-3,5 $\mathrm{mm}$ : Prospection continue dans le prolongement de l'axe antéropostérieur en période d'éclairement. Nage permanente avec constants changements de direction dans tous les plans tous les 0,5 à $2 \mathrm{~cm}$ de parcours. Propulsion et orientation par vibration caudale. Découverte de la proie à 1-2 $\mathrm{mm}$.

$3,5-4,5 \mathrm{~mm}$ : Début de prospection discontinue avec 2 maxima à $8-10 \mathrm{~h}$ ( $1^{\mathrm{re}}$ distribution) et $12-15 \mathrm{~h}$, ainsi qu'un $3^{\mathrm{e}}$ à $18-19 \mathrm{~h}$ lorsque les rations distribuées sont élevées. Prospection continue dans le cas contraire. Nage non prospective rectiligne ou curviligne sans arrêts. Nage prospective caractérisée par l'alternance de courtes trajectoires rectilignes de 1 à $3 \mathrm{~cm}$ et de périodes d'observation gauche-droite à l'arrêt, suivies de changements de direction. Propulsion par vibration caudale et orientation par action des pectorales. Découverte de la proie à $3-4 \mathrm{~mm}$.

4,5-13 $\mathrm{mm}$ : Prospection discontinue. Activité natatoire de base calme avec trajectoires longues, rectilignes ou légèrement curvilignes et mode de propulsion identique à celui décrit par WeIss (1974). Nage prospective identique à la précédente. Perception de la pro:e à $1-10 \mathrm{~cm}$ de distance.

13-20 $\mathrm{mm}$ : Prospection discontinue. Activité natatoire de base très calme se résumant à un maintien des coordonnées par petits bonds dus à 1 seul battement simultané des pectorales et de la caudale. Mode de chasse assimilable à un affût pélagique avec attaque rapide des proies qui passent et reprise immédiate de la position précédente. Activité natatoire et pros- 
pection classique en début de distribution d'aliment. Perception de l'aliment à $10-20 \mathrm{~cm}$.

$>20 \mathrm{~mm}$ : Prospection discontinue. Début des réactions rapides de groupe à la découverte de l'aliment. Début d'alimentation sur le fond ou à l'extrême surface. Nage caractéristique des juvéniles. Découverte de la proie à plus de $20 \mathrm{~cm}$, possibilité de conditionnement aux aliments inertes de type poudre ou paillettes flottant en surface.

\section{b) Sélectivité alimentaire}

L'étude des contenus stomacaux enseigne que dès les premiers jours d'alimentation, les larves sont zooplanctonophages et que leur spectre alimentaire est vaste tant sur le plan spécifique que dimensionnel. Tout comme pour d'autres sparidés (Kentouri \& Divanach, 1982), le marbré effectue un choix trophique qu'il exprime de deux façons : abandon après observation et régurgitation après ingestion.

Face à un plancton polyspécifique polydimensionnel, la sélectivité se traduit par des préférences alimentaires qui évoluent au cours de la croissance (tabl. 5 et 6). Les très jeunes poissons $(2,9-3,5 \mathrm{~mm})$ choisissent en premier lieu les ciliés (Strobilidium sp., Favella ehrenbergii et $F$. markusowskii) et les véligères de lamellibranches; puis les rotifères (Synchaeta vorax et Brachionus plicatilis sous sa forme marine naine) et ingèrent sporadiquement, les larves trochophores, les nauplii et meta nauplii de copépodes et de balanes. De 3,5 à $7,5 \mathrm{~mm}$, les préférences vont vers les rotifères mais les crustacés planctoniques sont déjà bien représentés dans le bol alimentaire alors que les ciliés, les trochophores et les véligères commencent à être délaissés. Au-delà de $7,5 \mathrm{~mm}$, les poissons choisissent les crustacés (copépodes, Artemia) et les rotifères sont à leur tour délaissés. A partir de $20 \mathrm{~mm}$, le régime s'enrichit d'organismes benthiques, copépodes, amphipodes, caprelles, larves d'annélides ainsi que d'algues filamenteuses non digérées qui peuvent représenter 30 p. 100 du volume stomacal total.

Cette sélectivité spécifique permet aux individus de découvrir et d'ingérer en proportion numérique élevée des catégories planctoniques très peu représentées dans le plancton distribué et inversement de négliger des formes qui y sont majoritaires (tabl. 6). Toutefois, de nombreuses catégories planctoniques de grande taille numériquement négligeables, représentent pourtant une fraction pondérale élevée du bol alimentaire (tabl. 6).

Les raisons de sélection sont nombreuses et pas toutes identifiées. Outre les critères spécifiques, ceux dimensionnels ou relatifs à l'origine des planctontes rentrent en ligne de compte ; soit :

- à l'intérieur d'une même catégorie systématique, la taille des proies est un critère de choix important (fig. 3). De 2,9 à $3,2 \mathrm{~mm}$, les larves peuvent ingérer toutes les classes de taille des populations de Strobilidium sp., Favella sp., Cephalodella sp., mais ne profitent que des fractions inférieures à $150 \mathrm{\mu m}$ des populations de trocophores, véligères, nauplii et Brachionus. De 4 à $6 \mathrm{~mm}$, toute la gamme de taille des nauplii et rotifères leur est accessible, mais les copépodes ne sont encore représentés que par leurs stades juvéniles ou inférieurs à $500 \mu \mathrm{m}$. Les nauplii d'Artemia salina sont ingérés à partir de $6 \mathrm{~mm}$ tandis que les formes adultes ne le seront pas avant $20 \mathrm{~mm}$. Entre 2,9 et $14 \mathrm{~mm}$ de taille, la largeur des proies ingérées augmente progressivement et présente une distribution très homogène (fig. $3 \mathrm{~B}$ ) mais reste le paramètre limitant l'ingestion. Par opposition, le spectre dimensionnel de la longueur des proies est très vaste et les 


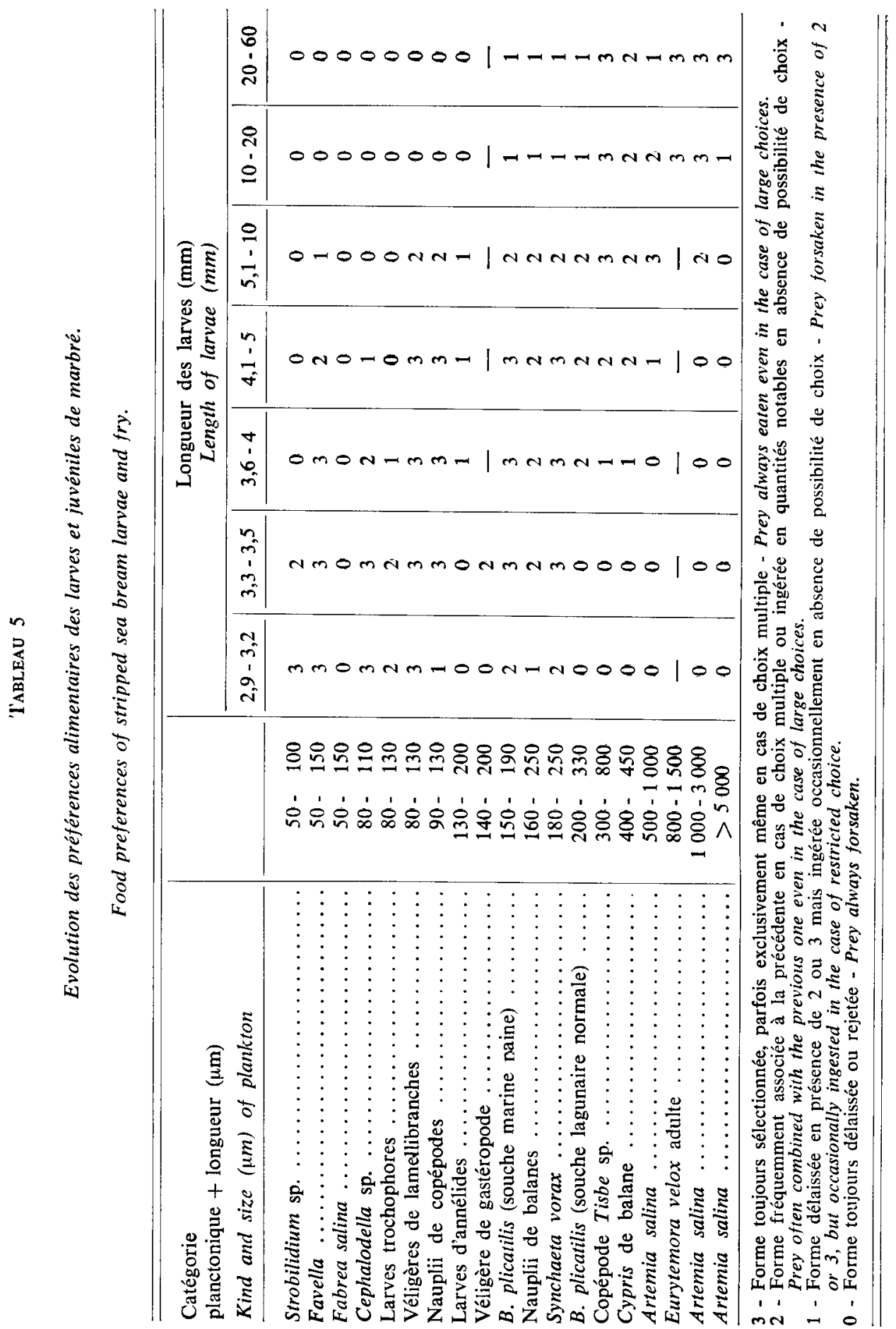




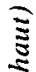

$m \mid$\begin{tabular}{ll|ll|ll|ll|ll|ll}
$m$ & 0 & 0 & 0 & $\stackrel{\infty}{f}$ & 0 & 0 & 0 & 0 & 0 & 0 & 0
\end{tabular}

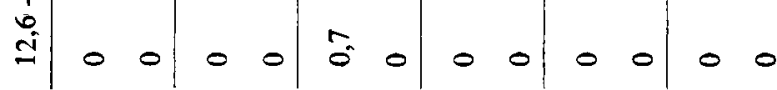

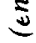

\begin{tabular}{l|ll|ll|ll|ll|ll|ll}
$\tilde{y}$ & 0 & 0 & 0 & 0 & \multirow{2}{*}{} & 0 & 0 & 0 & 0 & 0 & 0 & 0
\end{tabular}

華

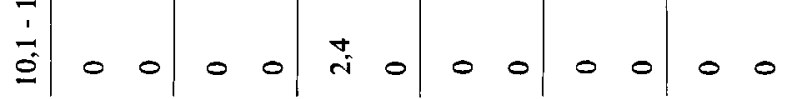

$\approx$

$\stackrel{\Xi}{\Xi}$

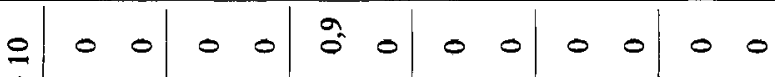

2

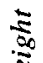

ธิข

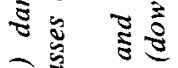

पบे

표

3

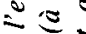

$\overline{8}$

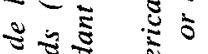

วิฐ

อ ะ

ระ ะ

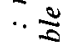

ว

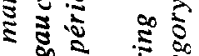

in

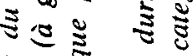

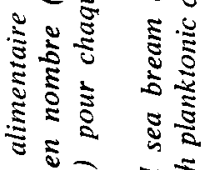

范

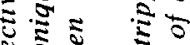

竞

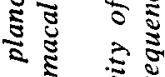

$\%$

造

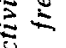

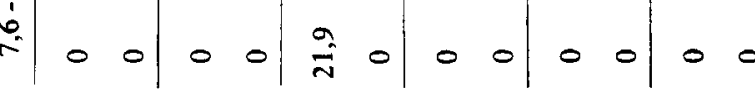

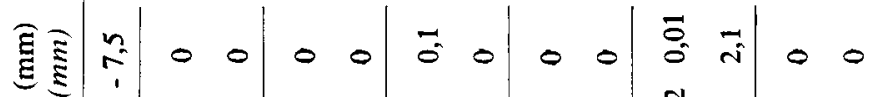

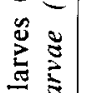

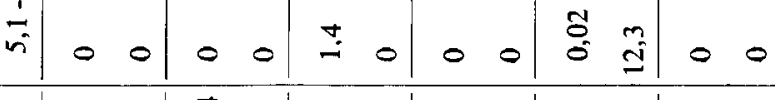

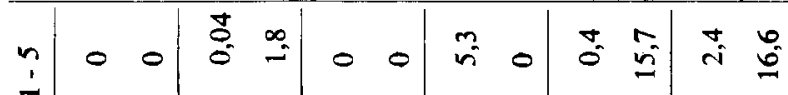

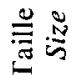

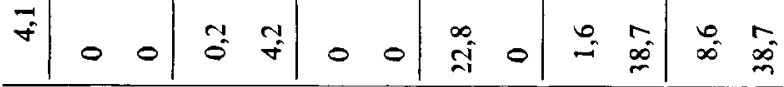

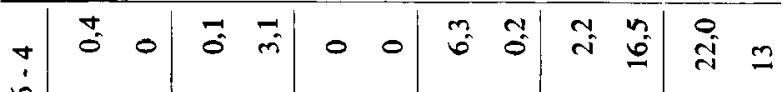

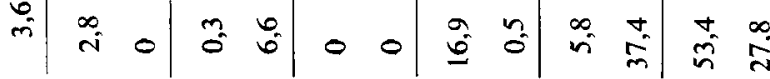

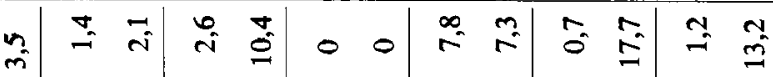

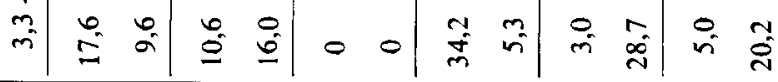

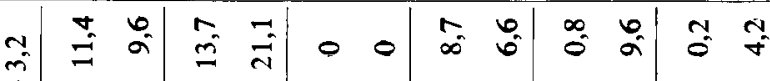

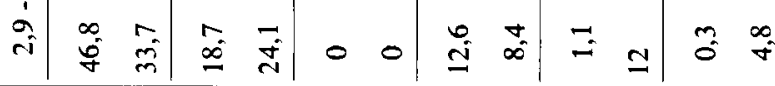

:

בั้

$\approx$

苋

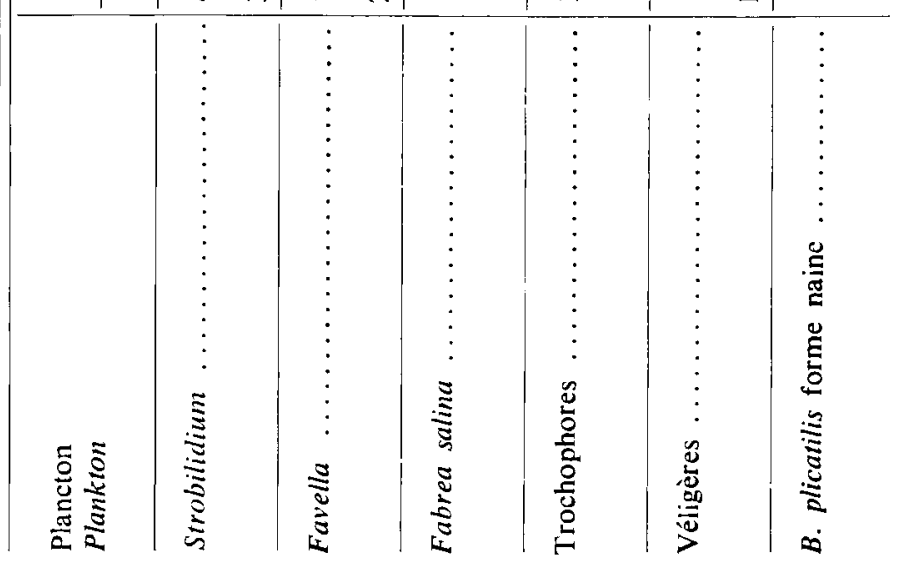




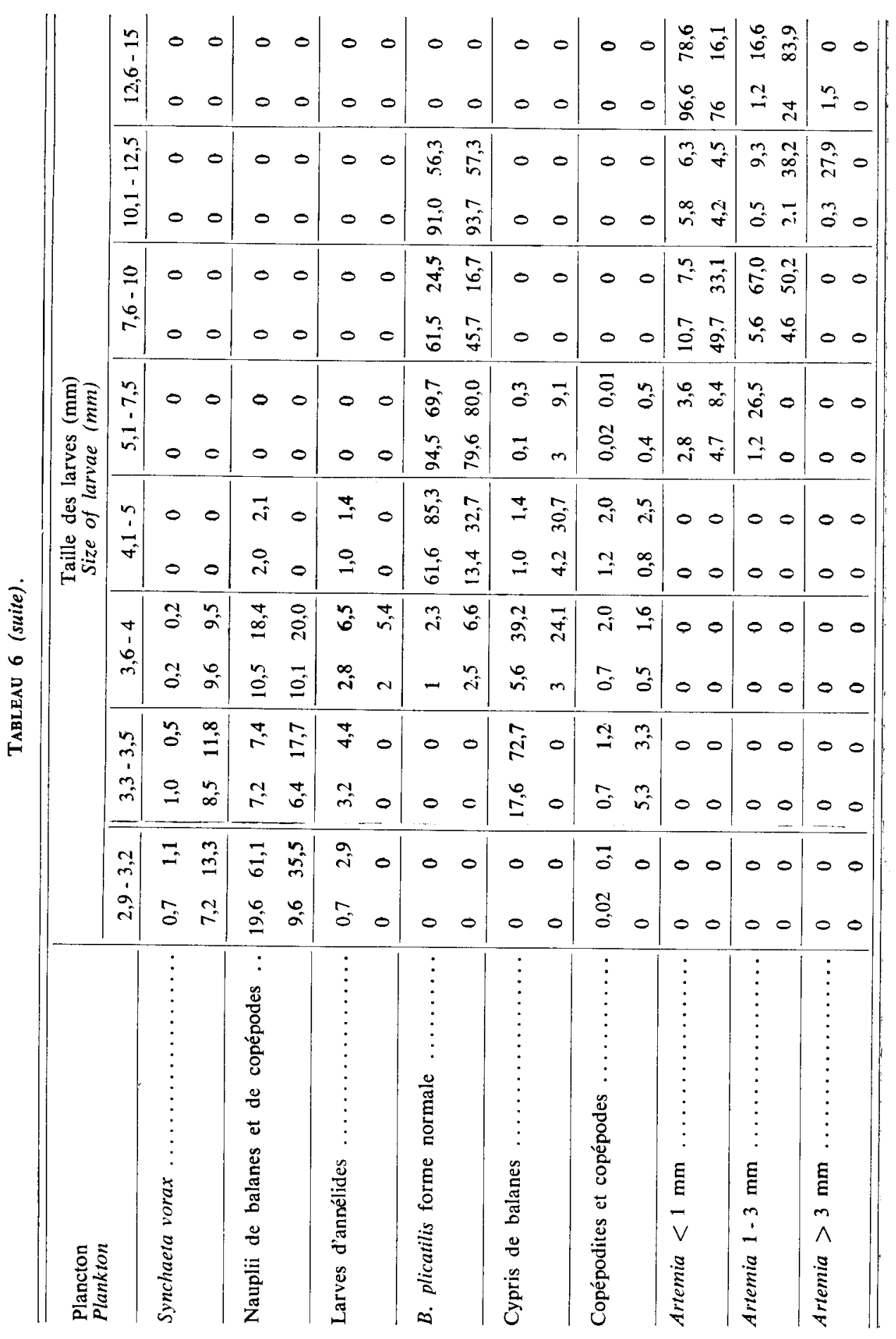


performances des larves sont bien supérieures aux valeurs moyennes calculées sur l'ensemble du contenu stomacal (fig. $3 \mathrm{C}$ ). Le phénomène est très net à partir de $10-15 \mathrm{~mm}$, lorsque les larves deviennent capables par le biais de régurgitations successives de «manipuler $\gg$ et d'orienter les proies avant de les avaler. Toutefois, tous les essais d'alimentation effectués avec du plancton de taille centrée sur les maxima possibles ont rapidement conduit à un affaiblissement des individus ;

- dans le cas de $B$. plicatilis, les individus d'origine marine restent longtemps des proies préférentielles alors que $B$. plicatilis provenant de zones de lagunage polluées est toujours une proie substitut qui n'est ingérée qu'occasionnellement en cas de choix multiple.

\section{c) Autres traits du comportement}

Dans nos conditions, l'espèce n'est ni agressive, ni cannibale, ni nécrophage. Les relations intraspécifiques se limitent à la formation de groupes ou d'essaims temporaires liés à des conditions trophiques à partir de $4,5 \mathrm{~mm}$ et parfois entre 15 et $20 \mathrm{~mm}$ à de courtes poursuites motivées par la possession d'une proie.

Les réactions aux agressions directes (toucher avec une aiguille ou essais de prélèvement) et indirectes (essentiellement siphonage et opérations de vidange des bassins) sont assez variées. Jusqu'à $8 \mathrm{~mm}$, elles sont isolées et se font dans le sens opposé à l'agression. Au-delà de $8 \mathrm{~mm}$, la peur conduit à des formations grégaires localisées au centre des bassins ( 8 à $13 \mathrm{~mm}$ ) ou près du fond (de 13 à $20 \mathrm{~mm}$ ). Ultérieurement, les réponses se diversifient : suivant le cas, les animaux plongent vers le fond et s'enfouissent dans le sédiment; ils sautent hors de l'eau ou ils fuient en éventail.

Le rhéotactisme positif commence à se manifester, sous forme d'une orientation corporelle, vers $3 \mathrm{~mm}$ et sous forme d'une remontée active du courant, qui permet d'éviter certaines zones d'aspiration, vers $4 \mathrm{~mm}$. Les conséquences appliquées de ces comportements se résument de la façon suivante :

— possibilité de siphonage des bassins avec des taux de perte inférieurs à 0,5 p. 100 par opération sans autre précaution que la prévention des turbulences (animaux compris entre 4 et $20 \mathrm{~mm}$ ). Au-delà de $20 \mathrm{~mm}$, les résultats sont identiques à condition de ne pas apeurer les animaux ou que le bassin soit exempt de sédiment ou d'algues;

- possibilité de vidanger une fraction importante du volume du bac, sans crépine de protection, avec un taux de perte inférieur à 0,5 p. 100 par opération à condition de ne pas apeurer les animaux $(50$ p. 100 du volume entre 4 et $10 \mathrm{~mm}, 90$ p. 100 au-delà).

2. Anomalies comportementales pendant l'essai $E_{2}$ (tabl. 2, 3 et 4)

Pendant l'essai $\mathbf{E}_{\alpha}$, l'évolution du comportement larvaire est différente de celle précitée :

- au $4^{\circ}$ jour, malgré une morphologie et une activité natatoire identiques à celles de leurs congénères, les larves ne se nourrissent pas;

- du $5^{\circ}$ au $9^{\prime \prime}$ jour, leur comportement de chasse s'exacerbe. La vitesse de nage, le volume prospecté, le nombre de proies observées et ingérées par jour sont supérieures aux valeurs trouvées dans l'essai 1, mais le schéma trophique est entaché de nombreux abandons après observations, de régurgitations après attaque et d'échecs; 
- du $10^{\circ}$ au $14^{\circ}$ jour, l'activité natatoire diminue, les individus reprennent progressivement les positions de repos caractéristiques des prélarves, leur longueur régresse et une mortalité massive est observée;

- ultérieurement, les rares survivants $(0,6$ p. 100$)$ se développent de façon parallèle à ceux élevés en bassin extérieur, mais une mortalité chronique s'installe. Elle est précédée de comportements spéciaux faisant alterner des phases d'hyperexcitation en position verticale, museau pointé à la surface ou contre les parois, suivies de longues périodes d'apathie en position de repos caractéristique des prélarves (45 ${ }^{\circ}$, tête en bas). Ces individus ne réagissent pas aux agressions et meurent en quelques jours dans un état de maigreur extrême.

La comparaison de lévolution des contenus stomacaux dans les deux essais montre (tabl. 2) une équivalence dimensionnelle et numérique initiale mais une différence de composition spécifique très marquée qui évolue dans l'essai 2 vers une réduction de la diversité et de la taille puis à une sélection de trochopores et véligères à faible vitesse de nage qui se fait au détriment des crustacés plus rapides.

\section{V. Discussion - Conclusions}

Malgré une taille de $1,7 \mathrm{~mm}$ à l'éclosion qui la classe parmi les plus petits sparidés jusqu'à présent étudiés (Kentourı \& Divanach, 1982), la larve de L. mormyrus présente des possibilités d'adaptation équivalents à ceux d'autres membres de la famille. Durant le stade prélarvaire, l'évolution du comportement moteur, l'apparition de la photosensibilité et l'échelonnement des modes de réactions aux stress suivent un chronodiagramme proche de celui établi pour Sparus auratus (Kentouri \& Divanach, 1982). La maitrise des réflexes de chasse survient lorsque toutes les étapes d'adaptation progressive décrites pour Diplodus sargus, D. vulgaris et $S$. auratus (Kentouri \& DivA$\mathrm{NACH}, 1982$ ) sont franchies.

Tout comme chez d'autres espèces, l'entrée dans la vie trophique peut être facilitée ou inhibée par les facteurs du milieu. Parmi eux, la taille des proies initiales est d'une importance capitale. La synthèse des travaux réalisés (YASUDA, 1960 ; YокотA et al., 1961 ; Detwyler \& Houde, 1970 ; Shirota, 1970 ; De Mendiola, 1974 ; Arthur, 1976) met en évidence une spécificité qui est confirmée par les résultats rapportés ici : la largeur maximale des proies ingérées par la larve de marbré en début de nutrition (2,9 à $3,2 \mathrm{~mm}$ de taille) est de $85 \mu \mathrm{m}$, alors que cette limite est de $100 \mathrm{\mu m}$ pour la larve de daurade longue de $4 \mathrm{~mm}$ à son entrée dans la vie trophique. Toutefois, ces différences initiales ne présument pas des possibilités ultérieures de l'animal qui sont régies par des lois spécifiques : à taille égale, les dimensions des proies ingérées par les larves de marbré sont nettement supérieures (fig. 3) à celles avalées par la daurade.

Si la présence des proies de taille adéquate permet au poisson d'élaborer rapidement les réflexes indispensables de prédateur, elle ne conduit pas pour autant à une croissance et une maturation comportementale correcte car les besoins nutritifs et le potentiel enzymatique de l'espèce doivent être pris en considération : les ciliés pélagiques totalement négligés en aquaculture marine sont parfaitement adaptés à la physiologie de $L$. mormyrus. Par opposition, les véligères, longues à digérer, nous semblent respon- 
sables de l'affaiblissement et de la mortalité observés chez les animaux de 3,6 à $4 \mathrm{~mm}$ de taille au cours de l'essai $\mathrm{E}_{2}$.

Bien que le résultat final soit le même (ralentissement de la croissance, résorption rapide des réserves lipidiques, mortalité), les défauts d'alimentation conduisent à des réactions comportementales différentes selon la taille des individus et les éthogrammes prévisionnels d'une mortalité se résument comme suit :

a) chez les animaux de 2,9 à $3,2 \mathrm{~mm}$ (entrée dans la vie trophique).

1. non alimentation le jour de la pigmentation des yeux;

2. prospection supranormale les jours suivants, parfois accompagnée d'une réplétion stomacale maximale, ou transfert de chasse vers les bulles d'air ;

3. régression de l'activité de chasse; attaque des particules ou proies immobiles ; réapparition des positions de repos caractéristiques des prélarves.

b) chez les larves mal nourries de 5 à $10 \mathrm{~mm}$ de longueur.

1. nage lente interrompue par des périodes de repos en position dorsale maintenue grâce à des efforts réguliers de réajustement (la larve a tendance à tomber en position $90^{\circ}$ tête en bas) ;

2. alternance de comportements de surexcitation, museau pointé contre les interfaces et de comportements régressifs d'apathie.

Dans chaque cas, l'apparition de la phase d'apathie correspond au \& point de non retour » tel qu'il est défini par Blaxter \& HeMPel (1963). Les autres phases, bien que réversibles, conduisent à un retard dans la croissance qui ne se rattrape pas et à une sensibilité du cheptel face à toute erreur alimentaire commise ultérieurement.

Ces observations qui vont de pair avec l'aptitude des larves à élaborer des comportements de protection adaptés aux circonstances, donnent à penser que sous des conditions compatibles avec leur physiologie, aucun déficit majeur ne peut être imputé au facteur «taille à l'éclosion». Par opposition, sous des conditions défectueuses, elles présentent plus rapidement et de façon plus aiguë les symptômes de la phase critique et, à ce titre, pourraient servir d'indicateurs biologiques.

Après le stade larvaire, les grands axes du comportement des juvéniles, tel que nous l'avons décrit, corroborent les observations effectuées en milieu naturel. L'enfouissement des juvéniles dans le sédiment après un stress est confirmé par SuAU (1970). L'apparition du caractère benthique vers $15 \mathrm{~mm}$ correspond aux observations de R.ANZI (1934), sur la présence d'individus de cette espèce le long de la côte. L'ingestion par les juvéniles de marbrés, de polychaetes, copépodes harpacticoïdes, amphipodes gammaridés et autres petits crustacés est également constatée par SuAU (1970) et Froglia (1977), mais l'ingestion d'algues filamenteuses non signalée pourrait être un biais lié à nos conditions d'élevage. 


\section{Summary}

\section{Contribution to the knowledge of behaviour and biology}

of stripped sea bream Lithognathus mormyrus larvae (Sparidae) in rearing conditions

Lithognathus mormyrus larvae were reared from hatching to fry stage in large tanks.

In polyspecific polydimensional breeding conditions including pelagic marine ciliates, the larvae grew and survived well (tabl. 4). Behavioural trends resembled those of other sparids (Kentouri \& DIVANaCH, 1982) ; feeding was always diversified, but mainly composed of 4 types of prey : from 2.9 to $3.5 \mathrm{~mm}$, ciliates and veligers; from 4 to $7 \mathrm{~mm}$, rotifers, up to $7 \mathrm{~mm}$, crustacea (tabl. 5 and 6).

When ciliates were not included in the ration, the early trophic phase was disturbed. Growth was low, mortality very high and survivors often entered a critical stage. After a supra normal period of prospection, activity and feeding decreased, the larvae exhibited prelarval rest postures and then died.

\section{Références bibliographiques}

Arthur D.K., 1976. Food and feeding of larvae of three fishes occurring in the California Current, Sardinops sagax, Engraulis mordax and Trachurus symmetricus. Fish. Bull. U.S., 74, 517-530.

Blaxter J.H.S., Hempel G., 1963. The influence of egg size on herring larvae. J. Cons. Int. Explor. Mer, 28, 211-240.

Cadenat J., 1937. Recherches systématiques sur les poissons littoraux de la côte occidentale d'Afrique, récoltés par le navire Président Théodore-Tissier au cours de sa $5^{\circ} \mathrm{croi}$ sière. Sparidae. Rev. Trav. off. Sci. tech. Pêches Marit., 10 (4), 464-469.

DE Mendiola R.G., 1974. Food of the larval anchoveta Engraulis ringens. In : J.H.S. Blaxter (ed.), The early life history of fish, Springer Verlag, New York, 227-285.

DetwYller R., Houde E.D., 1970. Food selection by laboratory reared larvae of the scaled sardine Harengula pensacolae (Pisces clupeidae) and the fry anchovy Anchovy mitchilli (Pisces engraulidae). Mar. Biol., 7, 214-222.

Divanach P., Kentouri M., 1983. Développement embryonnaire et larvaire du marbré Lithognathus mormyrus en élevage extensif ; accepté par Cybium, à paraître.

Froglia A., 1977. Feeding of Lithognathus mormyrus (L.) in central adriatic sea (Pisces, Sparidae). Rapp. Comm. Int. Mer Médit., 24 (5), 95-97.

Heldt H., 1926. Sur la résistance à l'asphyxie des principales espèces d'animaux marins. Applications à la tenue des aquariums et au transport des crustacés par bateau vivier. Publ. Stat. Océanogr. Salammbo, 2, 3-23.

Kentouri M., Divanach P., Paris J., 1981. Préférences alimentaires et comportement de la larve de daurade Sparus auratus face à du zooplancton congelé. Ann. Zootech., 30, 391-410.

Kentouri M., Divanach P., 1982. Différences et similitudes dans la genèse des comportements locomoteur et trophique des stades prélarvaires de Sparus auratus, Diplodus vulgaris, Diplodus sargus. Aquaculture, 27, 355-376.

Kentouri M., Divanach P., 1982. Action des facteurs du milieu sur le comportement de la larve de daurade Sparus auratus en élevage extensif. Rapport interne, Station de Biologie Marine et Lagunaire, Sète, 45 p.

Lissia Frau A.M., 1968. Le manifestazioni della sessualita negli Sparidi (teleostei perciforme). Studi Sassaressi, 46 (2), 243-261.

Lozano Cabo F., 1969. La fauna ictiologica del Mar Menor. 1. Generalidades y claves de determinacion de las especies. Boll. Inst. Espanol de Oceanogr., 138, 3-47. 
Lozano y Rey L., 1952. Peces fisoclistos. Sparidae. Memorias de la Real Academia de Ciencias, 14, 120-201.

Moreau E., 1881. Histoire naturelle des poissons de la France. Sparidae, 3, 1-62. Masson ed.

Phisalix M., 1931. Action des venins de vipère aspic et de ceraste cornu sur quelques poissons marins. Publ. Stat. Océanogr. Salammbo, 25, 3-20.

RanZI S., 1934. Uova, larve e stadi giovanili dei teleostei. Sparidae, Fauna e Flora del Golfo di Napoli. Monografia, 38, 332-382.

Shrrota A., 1970. Studies on the mouth size of Fish Larvae. Bull. Jap. Soc. Sci. Fish., 36 (4), 353-368.

Smith J.L.B., 1953. The sea fishes of Southern Africa. Sparidae, 265-278. Central News Agency LTD ed.

Suau P., 1955. Contribucion al estudio de la herrera (Pagellus mormyrus L.) (II) especialmente de la sexualidad. Inv. Pesq., 1, 59-66.

SuAu P., 1970. Contribucion al estudio de la biologia de Lithognathus (= Pagellus) mormyrus L. (Peces esparidos). Inv. Pesq., 34 (2), 237-265.

Varagnolo S., 1963. Calendario di comparse di uova pelagiche di teleostei marini nel plancton di Chioggia. Arch. Oceanogr. limnol., 13 (2), 249-281.

WEISs D., 1974. Energetic advantages of burst swimming of fish. J. Theor. Biol., 48, 215-229.

Yasuda F., 1960. The feeding mechanism in some carnivorous fishes. Rec. Oceanogr. Works Jap., 5 (2), 153-160.

Yokota T., Toriyama M., Kanai F., Nomura S., 1961. Studies on the feeding habit of fish. Rep. Nankai Reg. Fish. Res. Lab., 14, 234 p.

Zuniga L.R., Suau P., 1967. Nota sobre la presencia de los copepodos parasitos Peniculus fistula y Lernaeolophus sultanus sobre dos nuevos huespedes Lithognathus mormyrus y Boops boops. Inv. Pesq., 31 (3), 485-487. 